\title{
Special Issue on Semantic Web
}

\author{
Birte Glimm $^{1} \cdot$ Heiner Stuckenschmidt ${ }^{2}$
}

Received: 18 January 2016/ Accepted: 15 February 2016/Published online: 25 February 2016

(C) Springer-Verlag Berlin Heidelberg 2016

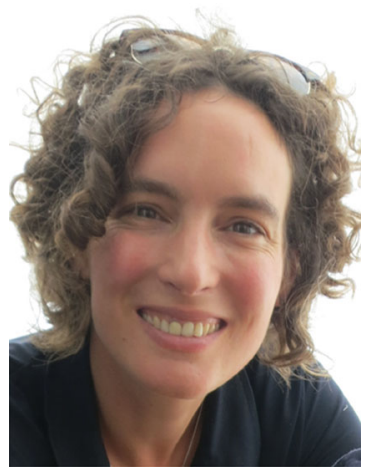

Birte Glimm

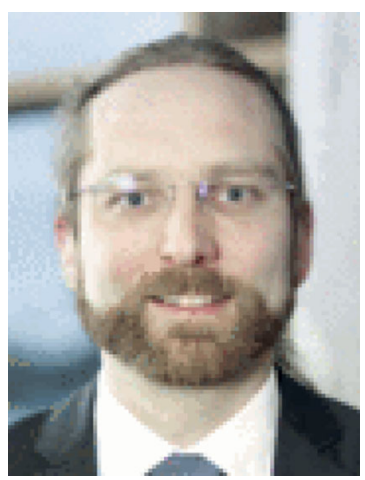

Heiner Stuckenschmidt

Birte Glimm

birte.glimm@uni-ulm.de

Heiner Stuckenschmidt

heiner@informatik.uni-mannheim.de

1 Institute of Artificial Intelligence, University of Ulm, 89081 Ulm, Germany

2 Chair of Artificial Intelligence, University of Mannheim, 68161 Mannheim, Germany
Fifteen years ago Tim Berners-Lee, James Hendler, and Ora Lassila formulated the idea of a Semantic Web that extends the standard Web by giving information well-defined and computer-processable meaning with the goal of enabling computers and people to work in cooperation. Since then, this idea led to many developments. For example, the crowedsourced community effort DBpedia makes a large part of the free encyclopedia Wikipedia available in a structured and machine processable format. The English version of DBpedia alone describes 4.58 million things $(1,445,000$ persons, 735,000 places, 411,000 creative works, 241,000 organizations, 251,000 spe-

cies, and 6,000 diseases) and 125 localized versions are further available. The developments are, however, not limited to the area of the (Semantic) Web itself. For example, the Systematized Nomenclature of MedicineClinical Terms (SNOMED CT), which is considered to be the most comprehensive, multilingual clinical healthcare terminology in the world, is formulated in the Description Logic EL ++ . This Description Logic is the basis of OWL 2 EL, i.e., one of the standardized fragments of the Web Ontology Language (OWL) and the terminology is readily available in the typical Semantic Web serialization formats.

While initially the standardization and research efforts were focussed on very expressive knowledge representation formalisms with (highly) complex algorithms for deriving entailed knowledge, recent trends like the Linked Open Data (LOD) initiative focus more on providing openly available, structured and interlinked datasets for which automated reasoning plays a less important role. Following the trend towards less expressive knowledge representation formalisms and building on research results about tractable reasoning procedures for such formalisms, the second version of OWL explicitly defines three socalled profiles that allow for implementing tractable automated reasoning procedures.

A key technology for the successful use of knowledge within the Semantic Web is a widely adopted query language. This role has been taken by SPARQL over the past years, which is by now a sophisticated, W3C standardized query language with dedicated support for handling data in the resource description framework (RDF), the fundamental data model used in the Semantic Web.

Within this special issue, three survey articles introduce readers to the Semantic Web: Glimm and Stuckenschmidt summarize the standardization efforts in this area 
and look at the development of research trends and methodologies within the last 15 years; the survey by Peinl takes a more practical point of view and looks at tools and adoption of Semantic Web technologies in corporations; finally, Klusch et al. describe how semantic technologies allow for a high-precision search and an automated composition of Web services to build intelligent service-based applications.

Linked Open Data and, in particular, its benefits for other sciences is a topic taken up by four articles in this special issue. Zapilko et al. discuss challenges and solutions for interlinking domain-specific information and linking social science data to other LOD datasets. Posch et al. present a system for creating and visualizing probabilistic semantic links between concepts in a thesaurus and classes in a classification system and show how their system can be used to link two knowledge organization systems in the social sciences domain. Latif at al. then show how library science can benefit from using Linked Data. The research project SEPAL introduced by Scheglmann et al. addresses the practical challenge of programming applications against LOD.

Ontologies can not only be used to give a well-defined meaning to terms. Alsubait et al. show how knowledge captured in an ontology can be used to generate multiple choice questions of a controlled difficulty that can then be used in examinations or practice material for students. Over the recent years, ontologies are also more and more used to enrich the data in (relational) databases, i.e., they are used as expressive schemas. Krötzsch and Rudolph present different approaches for ontology-based query answering, i.e., where the schema in the form of an ontology is used to enrich the answers to queries over the data. Möller et al. also consider query answering over ontologies, but they look at how abductive query answering can be implemented, where not all query atoms have to be satisfied by a query answer. For unfulfilled query atoms hypothesis are generated about how the query atom could be satisfied.

Taking up the challenge of learning the semantic relatedness between words, Niebler at al. discuss how this can be done based on navigation data for Wikipedia.

Finally the interviews with Prof. Dr. Rudi Studer and Prof. Dr. Ian Horrocks retrace the developments in the Semantic Web over the last 15 years, highlight interesting applications and identify possible research trends for the years to come.

This special issue is the result of the efforts of many persons. Special thanks go to the authors for their contributions and their help in putting this special issue together, our interview partners for taking the time to share their opinions and experiences about this area, and to the $\mathrm{KI}$ team, in particular the editor in charge of this issue, Ubbo Visser ( University of Miami), for his excellent support.

Birte Glimm and Heiner Stuckenschmidt

\section{Content}

\subsection{Technical Contributions}

- Birte Glimm and Heiner Stuckenschmidt: 15 years of semantic web-an incomplete survey

- René Peinl: Semantic web-state of the art and adoption in corporations

- Matthias Klusch, Patrick Kapahnke, Stefan Schulte, Freddy Lecue, and Abraham Bernstein: Semantic web service search: a brief survey

- Atif Latif, Ansgar Scherp, and Klaus Tochtermann: LOD for library science: benefits of applying linked open data in the digital library setting-retrospects and research topics

- Benjamin Zapilko, Johann Schaible, Timo Wandhöfer, and Peter Mutschke: Applying linked data technologies in the social sciences

- Thomas Niebler, Daniel Schlör, Martin Becker, and Andreas Hotho: Extracting semantics from unconstrained navigation on wikipedia

- Markus Krötzsch and Sebastian Rudolph: Is your database system a semantic web reasoner?

- Ralf Möller, Özgr Özçep, Volker Haarslev, Anahita Nafissi, and Michael Wessel: Abductive conjunctive query answering w.r.t. ontologies

\subsection{Research Projects}

- Tahani Alsubait, Bijan Parsia, and Uli Sattler: Ontology-based multiple choice question generation

- Stefan Scheglmann, Martin Leinberger, Thomas Gottron, Steffen Staab, and Ralf Lämmel: SEPAL—schema enhanced programming for linked data

\subsection{System Descriptions}

- Lisa Posch, Philipp Schaer, Arnim Bleier, and Markus Strohmaier: A system for probabilistic linking of thesauri and classification systems

\subsection{Interviews}

- Heiner Stuckenschmidt: Interview mit Prof. Dr. Rudi Studer

- Birte Glimm: Interview with Prof. Dr. Ian Horrocks 


\section{Service}

\subsection{Conferences}

- Semantic Web Track at the International World Wide Web Conference (WWW)-Core Ranking A+-in 2016: 25th International World Wide Web Conference (WWW 2016), April 11 to 15, 2016 in Montreal, Canada http://www2016.ca/

- AI and the Web Track at the AAAI Conference on Artificial Intelligence (AAAI) Core Ranking A+ - in 2016: 30th AAAI Conference on Artificial Intelligence (AAAI 16), February 12 to 17, 2016 in Phoenix, Arizona, USA http://www.aaai.org/Conferences/AAAI/ aaai16.php

- International Semantic Web Conference (ISWC) Core Ranking A - in 2016: $15^{\text {th }}$ International Semantic Web Conference (ISWC 16), October 17 to 21, 2016 in Kobe, Japan http://iswc2016.semanticweb.org/

- Extended Semantic Web Conference (ESWC) Core Ranking A - in 2016: $13^{\text {th }}$ Extended Semantic Web Conference (ESWC 16), May 29th to June 2nd, 2016 in Anissaras, Crete, Greece http://2016.eswc-conferences. org/

\subsection{Organizations}

- World Wide Web Consortium (W3C) http://www.w3. org/

- Semantic Web Science Association (SWSA) http:// swsa.semanticweb.org/

\subsection{Journals}

- Journal of web semantics (Elsevier)

- International journal on semantic web and information systems (IGI Global)

- Semantic web journal (IOS Press)

- Journal on data semantics (Springer)

- Transactions on the web (ACM)

\subsection{Books}

- Grigoris Antoniou, Paul Groth, Frank van Harmelen, and Rinke Hoekstra: A Semantic Web Primer. The MIT Press, 3rd Edition (2012)

- Dean Allemang and Jim Hendler: Semantic Web for the Working Ontologist. Morgan Kaufmann, 2nd Edition (2011)
- Tom Heath and Christian Bizer: Linked Data-Evolving the Web into a Global Data Space. Morgan \& Claypool Publishers (2011)

- Pascal Hitzler, Markus Krötzsch, Sebastian Rudolph: Foundations of Semantic Web Technologies, Chapman \& Hall/CRC (2009)

- Steffen Staab and Rudi Studer: Handbook on Ontologies. Springer (2009)

- Liyang Yu: A Developer's Guide to the Semantic Web. Springer (2015)

- Toby Segaran and Colin Evans: Programming the Semantic Web. O'Reilly Media (2009)

- Bob DuCharme: Learning SPARQL. O'Reilly Media; 2nd Edition (2013)

\subsection{Summer Schools}

- Semantic Web Summer School (SSSW) http://sssw. org/

- Reasoning Web Summer School http://reasoningweb. org/

\subsection{Software}

Overviews on tools are available from:

- http://semanticweb.org/wiki/Tools.html

- http://www.w3.org/2001/sw/wiki/Tools

- http://www.mkbergman.com/291/comprehensive-list ing-of-250-semantic-web-tools-updated/

\subsection{Web-Sites}

- http://semanticweb.org/

- Semantic Web Interest Group http://www.w3.org/2001/ sw/interest/

- W3C DATA ACTIVITY Building the Web of Data http://www.w3.org/2013/data/

\subsection{Mailing-Lists}

- Semantic Web Interest Group (SW IG) semanticweb@w3.org

- Linking Open Data project public-lod@w3.org

- A discussion list affiliated with SemanticWeb.org semanticweb@yahoogroups.com 